\title{
Dynamic Stress Drop of Recent Earthquakes: Variations within Subduction Zones
}

\author{
LARRY J. RUFF ${ }^{1}$
}

\begin{abstract}
Stress drop is a fundamental parameter of earthquakes, but it is difficult to obtain reliable stress drop estimates for most earthquakes. Static stress drop estimates require knowledge of the seismic moment and fault area. Dynamic stress drop estimates are based entirely upon the observed source time functions. Based on analytical formulas that I derive for the crack and slip-pulse rupture models, the amplitude and time of the initial peak in source time functions can be inverted for dynamic stress drop. For multiple event earthquakes, this method only gives the dynamic stress drop of the first event. The Michigan STF catalog provides a uniform data base for all large earthquakes that have occurred in the past four years. Dynamic stress drops are calculated for the nearly 200 events in this catalog, and the resultant estimates scatter between 0.1 and $100 \mathrm{MPa}$. There is some coherent tectonic signal within this scatter. In the Sanriku (Japan) and Mexico subduction zones, underthrusting earthquakes that occur at the up-dip and down-dip edges of the seismogenic zone have correspondingly low and high values of stress drop. A speculative picture of the stress state of subduction zones emerges from these results. A previous study found that the absolute value of shear stress linearly increases down the seismogenic interface to a value of about $50 \mathrm{MPa}$ at the down-dip edge. In this study, the dynamic stress drop of earthquakes at the up-dip edge is about $0.2 \mathrm{MPa}$, while large earthquakes at the down-dip edge of the seismogenic plate interface have dynamic stress drops of up to $5 \mathrm{MPa}$. These results imply that: (1) large earthquakes only reduce the shear stress on the plate interface by a small fraction of the absolute level; and thus (2) most of the earthquake energy is partitioned into friction at the plate interface.
\end{abstract}

Key words: Stress drop, rupture, seismogenic zone, source time functions, subduction, friction.

\section{Introduction}

Earthquakes reduce stress over most of the fault area, hence stress drop is a fundamental parameter of earthquakes. Unfortunately, it is difficult to reliably estimate stress drop; thus it is determined only in special studies of particular earthquakes. This lack of uniform treatment of earthquakes can be excused because one of the key tenets of seismology is that stress drop is "approximately constant" for earthquakes of all types and sizes (KANAMORI and ANDERSON, 1975; ScHOLZ,

\footnotetext{
${ }^{1}$ Department of Geological Sciences, University of Michigan, Ann Arbor, MI 48109, U.S.A.
} 
1990). In detail, "approximately constant" means that stress drop estimates typically fall in the range of 1 to 100 bars $(0.1$ to $10 \mathrm{MPa})$, with an occasional report of much higher values. Given the fundamental importance of stress drop to earthquake physics, seismologists must make progress on two fronts: (1) systematically estimate stress drop for all seismicity above some magnitude threshold; and (2) provide more reliable and consistent stress drop estimates such that we can extract information from within the factor of one hundred variation in current estimates. In this paper, I show that the dynamic stress drop for the initial rupture process can be reliably determined from source time functions, and I show results for large earthquakes that occurred in the past four years. These preliminary results do not reduce the scatter in stress drop estimates, but we do see some structure within the "approximately constant" stress drop. In particular, I focus on underthrusting earthquakes in subduction zones and find some evidence for systematic variation in the dynamic stress drop between events at the down-dip and up-dip edges of the seismogenic zone.

\section{Static Stress Drop}

Static stress drop is the simplest measure of the overall reduction in shear stress due to slip on the fault zone. It is the difference between the average shear stress on the fault zone before and after the earthquake (Fig. 1). Since the stress drop of real earthquakes varies across the fault area, the overall static stress drop is a slipweighted average of the spatially variable stress drop. Seismologists typically use simple constant stress drop models to estimate earthquake stress drops. Regardless of the details of fault geometry and slip distribution, the basic formula for stress drop is:

$$
\Delta \sigma_{s t}=c \mu \frac{D}{L}
$$

where $D$ is the average slip over the faulted area $(A), L$ is the characteristic length of the fault area, $\mu$ is the elastic shear modulus, and $c$ is a geometric constant that is close to one if $L$ is properly chosen. Since seismic moment $\left(M_{0}\right)$ for most large earthquakes can be reliably determined from seismic waves, rewrite the above equation as:

$$
\Delta \sigma_{s t}=c \mu \frac{D A}{L A}=c \frac{M_{0}}{L A} .
$$

This formula shows that we need three quantities to calculate stress drop: a measurement of the seismic moment, some estimate of the fault area $(A)$, and then some appropriate choice for the characteristic fault dimension. While the choice of $L$ presents an interpretational problem, it is the estimation of $A$ that presents 
practical difficulties and introduces large errors into stress drop estimates. Most estimates of $A$ are based on drawing a rectangle or ellipse around the aftershock area. This technique is trustworthy for great earthquakes with fault zones more than $100 \mathrm{~km}$ across, but its reliability decreases as fault dimensions decrease toward the epicenter location accuracy and as the number of aftershocks decreases. Since we expect the fault area of magnitude 7 earthquakes to have a characteristic dimension of about $10 \mathrm{~km}$, this aftershock technique is limited to just great earthquakes if we desire a uniform global study.

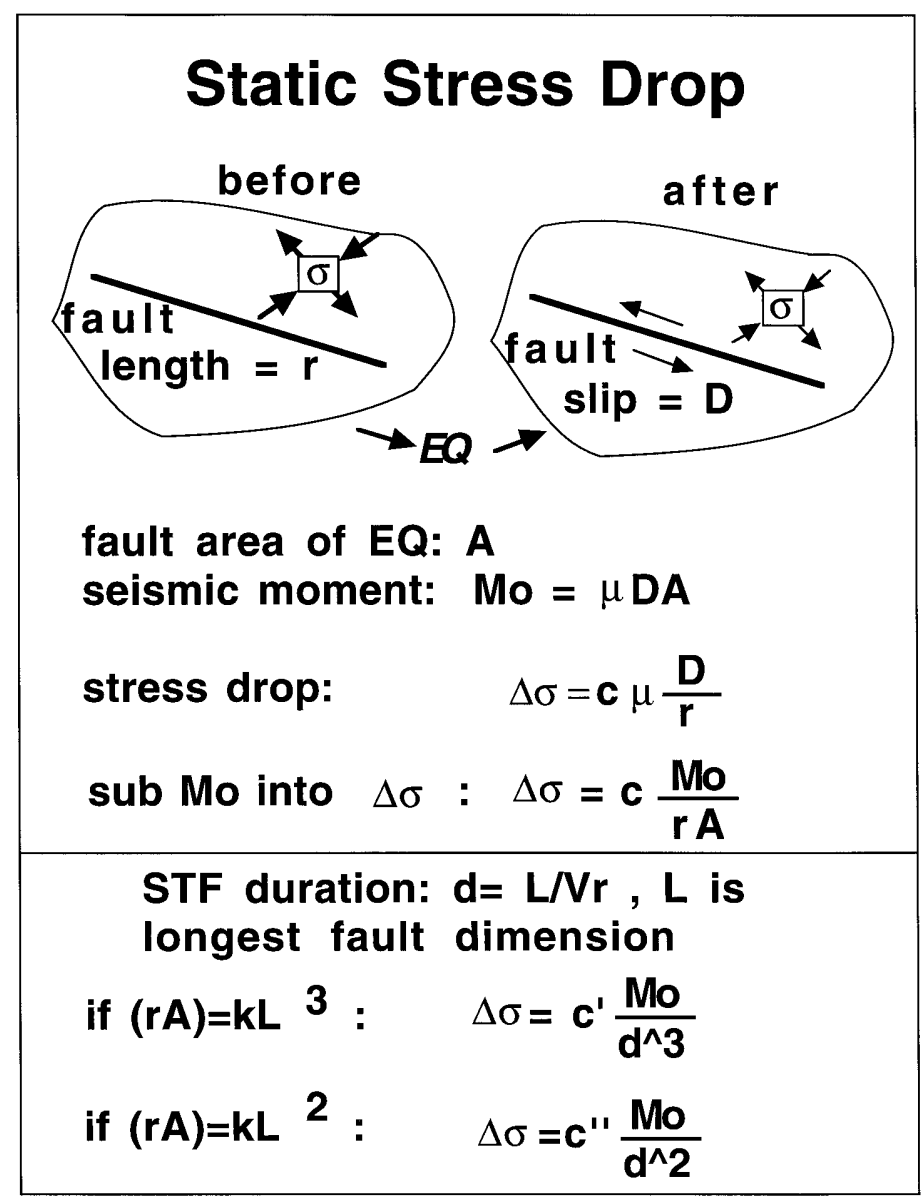

Figure 1

Basic definition and estimation of static stress drop. Upper part shows that estimates of static stress drop depend on seismic moment and fault area. It is common to use source time function duration to estimate $L$, the longest fault dimension. Lower part shows two possible choices for the scaling law, TANIOKA and RUFF (1997) find an empirical scaling relation that is an average of the two shown here. 
From a theoretical perspective, the best way to determine fault area is to use geodetic studies of the direct static deformations around the source area. In detail, there are interpretational difficulties due to the non-uniqueness of geodetic inversions, but the larger problem has been lack of geodetic monitoring of all the seismic regions of the world. As more GPS surveys are performed around the world, the number of earthquakes with geodetic control will increase in the future. The advent of tsunami seismology opens a new avenue for geodetic control of large earthquakes that occur beneath the ocean (SATAKE, 1995).

It is possible to extract the fault length from source time functions. If a large earthquake ruptures a long fault zone in a unilateral fashion, the directivity seen in the source time functions from azimuthally-distributed stations can be quantitatively exploited to determine the longer fault dimension. We usually use other means to estimate the shorter fault dimension. This rupture process method is time-consuming and difficult, and produces reliable results for just a few earthquakes.

We now turn to the most imprecise indicator of fault area, yet has proven to be the most useful method: some measure of rupture duration combined with assumptions of rupture velocity and fault geometry (lower part of Fig. 1). In the simplest case, we assume a circular fault geometry with radius $R$, and fixed rupture velocity, $V r$, with some typical value, say 2 or $3 \mathrm{~km} / \mathrm{s}$. Then, if we can extract the source time function from seismograms, we can interpret the duration, $d$, to be the rupture time: $d=R / V r$. Since the characteristic fault dimension for circular faults is $R$, and $c$ is $(7 \pi / 16)$, Equation (1) becomes:

$$
\Delta \sigma_{s t}=\frac{7}{16} \frac{M_{0}}{(d V r)^{3}} .
$$

Estimates of $\Delta \sigma_{s t}$ can be quite uncertain due to either interpretational problemsi.e., the rupture time may actually be $d / 2$, which would introduce a factor of 8 error in Equation (2); or the assumed value of $V r$ may be wrong. Also, perhaps the geometry of rupture is better approximated by a semi-circle, or quarter-circle, which would introduce a factor of 4 error. Finally, as depicted in Figure 1, a different assumption for fault geometry can change the functional dependence from $d^{-3}$ to $d^{-2}$.

\section{Dynamic Stress Drop}

There is no one universal definition of the dynamic stress drop because the space-time history of stress drop that is radiating waves can be quite variable. Indeed, any single portion of the fault plane probably has variable stress drop

during the time that it is slipping. Since it is impossible to reliably invert waves to determine the complete space-time history of dynamic stress drop, we must be 
satisfied with various space-time averages of the dynamic stress drop. The simplest view is that dynamic stress drop is constant over the space-time window that the fault is slipping. Since the final stress can change after a particular fault portion stops slipping, the static stress drop of that portion could be quite different than the time-averaged dynamic stress drop. This potential mismatch between dynamic and static stress drop addresses one of the key questions of earthquake rupture physics, as detailed below.

There is a simple connection between an idealized form of the dynamic stress drop and an observable kinematic parameter, the slip velocity (see, e.g., BRUNE, 1970; or discussions in AKI and RichaRds, 1980; and KASAHARA, 1981). If the rupture front could propagate at infinite velocity to unzip the entire fault plane, then the slip fault velocity is:

$$
\frac{d D}{d t}=\dot{D}=c \beta \frac{\Delta \sigma_{d y}}{\mu},
$$

where $\beta$ is the source region shear velocity and $\Delta \sigma_{d y}$ is the dynamic stress drop. We must construct some model for rupture front behavior to estimate $\Delta \sigma_{d y}$ based on the above equation. The simplest model is to assume that a circular fault grows over duration $d$ with constant rupture velocity $V r$, and that all parts of the fault have a slip velocity history that is zero before arrival of the rupture front, then $\dot{D}$ jumps to the above value and remains at that slip velocity. For this case-or any case in which the slip history of every place on the fault has the same time history that is shifted in time due to the rupture front - we can write the overall moment rate history as the convolution of the slip velocity function with the area growth rate function:

$$
\begin{aligned}
\dot{M}(t) & =\mu \dot{D}(t) * \dot{A}(t)=c \mu \beta \frac{\Delta \sigma_{d y}}{\mu} \int_{0}^{t} 2 \pi V r^{2} t^{\prime} d t^{\prime}, & & \text { for } 0 \leq t \leq d \\
& =c \mu \beta \frac{\Delta \sigma_{d y}}{\mu} \pi V r^{2} t^{2}, & & \text { for } 0 \leq t \leq d .
\end{aligned}
$$

The shape of this $\dot{M}(t)$ function (the source time function) is a growing quadratic function until a time of $d$. Of course, a complete rupture model must also specify when and how the fault stops slipping, but we ignore that aspect now. If we look at the value of $\dot{M}(t)$ at $t=d$, we have:

$$
\dot{M}(t=d)=c \mu \beta \frac{\Delta \sigma_{d y}}{\mu} \pi V r^{2} d^{2},
$$

and we then solve for $\Delta \sigma_{d y}$ in terms of the observed quantities of $\dot{M}(d)$ and $d$ :

$$
\Delta \sigma_{d y}=\frac{1}{c \pi \beta V r^{2}} \frac{\dot{M}(t=d)}{d^{2}}
$$


Rewrite $V r$ in terms of $\beta$ as $V r=f \beta$. Then the above equation is:

$$
\Delta \sigma_{d y}=\frac{1}{c \pi \beta^{3} f^{2}} \frac{\dot{M}(t=d)}{d^{2}} .
$$

Equation (3) shows that estimates of the dynamic stress drop are based on values taken from source time functions. Equation (3) displays some of the basic features that we will see in dynamic stress drop formulas for the more complicated rupture models considered in the next section. In particular, Equation (3) shows that dynamic stress drop depends on the observed quantity of the peak moment rate divided by the square of the time that this peak value is obtained. Also, the only dependence on source region parameters is $\left(1 / \beta^{3}\right)$, and the unknown rupture velocity enters as the dimensionless ratio $f$.

The above "Brune-type" dynamic stress drop model offers an excellent introduction to the topic, but now we must specify more complete rupture models such that the fault "heals" and fault slip eventually stops. In addition, we should try to find a dynamic rupture model that produces a final slip distribution compatible with some static stress drop.

\section{Cracks that Start and Stop}

Basic crack mechanics show that a propagating crack tip with a constant rupture velocity and stress drop produces a slip velocity function with a square-root singularity at the crack tip (see, e.g., AKI and RichARDS, 1980). Thus, a more realistic description of fault slip velocity must use the $t^{-1 / 2}$ function, as inset at top left of Figure 2, rather than the step function of the basic "Brune-type" model. The dynamic stress drop appears in the scaling of the $\left(t^{-1 / 2}\right)$ function. In detail, there is a complementary square-root singularity in the stress just ahead of the crack tip, hence there must be a small break-down zone around the crack tip where both the stress and slip velocity remain finite (AKI and RichARDS, 1980). Since source time functions are integrals of the slip velocity function, these alterations at the crack tip have no discernible effect. The rupture models discussed below use the $\left(t^{-1 / 2}\right)$ functional dependence of slip velocity, and the rupture starts at a point and expands with a circular rupture front. As seen in the above section, use of an expanding rupture front ensures a source time function that begins at zero and increases with time.

The other critical aspect of a dynamic rupture model is how the crack heals. Basic crack mechanics does not give us a simple universal behavior; we must invent different scenarios on how we think earthquake rupture fronts and fault slip stop. While it may be reasonable to suppose that most earthquakes start with a circular or elliptical rupture front, it is clear that rupture front geometry changes - at least for large earthquakes. Since fault areas for the largest strike-slip and underthrusting 


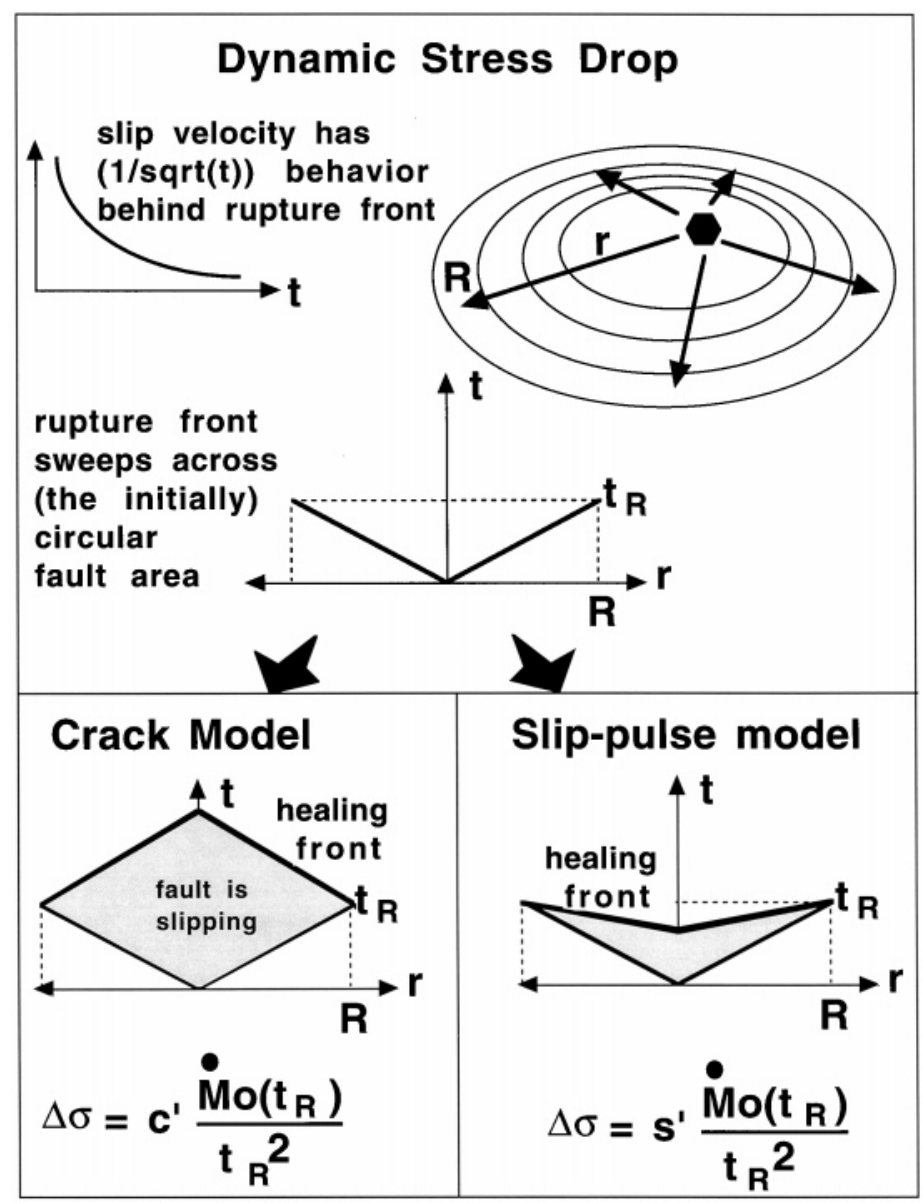

Figure 2

Graphical definition of the two dynamic rupture models derived in this paper. Any dynamic model must specify the slip velocity function and the behavior of the rupture and healing fronts. Both models use a $t^{-1 / 2}$ slip velocity function and a circular rupture front that expands with constant velocity out to $R$ (upper part). The models differ in the behavior of the healing front. For the crack model, the healing front propagates back across the fault after rupture termination at $R$, while for the slip-pulse model, a healing front initiates at $r=0$ and spreads out to eventually catch and stop the rupture front. The equations in lower part show that the dynamic stress drop for both models has a similar dependence on the parameters extracted from source time functions (see text), but that the $c^{\prime}$ and $s^{\prime}$ factors are different.

events tend to be elongated in the horizontal direction, the expanding circular 2-D rupture front must switch to a bilateral or unilateral 1-D rupture front when it reaches the fault width. Simple kinematic rupture models show that this switch from 2-D to 1-D rupture geometry is reflected in the source time functions (hereafter: STFs) by a drop in the moment rate (e.g., RUFF and KANAMORI, 1983). Thus, the relevance of an expanding circular rupture front to earthquakes is in 
doubt after the time of the initial peak value of the STF. For those STFs that have a multiple-event character, we can only estimate the dynamic stress drop for the first subevent.

Finally, we must consider how the fault slip stops. This healing process is one of the great mysteries about earthquakes. The two end-member views have been referred to as the "crack" and the "slip-pulse" models. In the crack model, once a point on the fault begins to slip, it continues to follow the $t^{-1 / 2}$ slip velocity function until the rupture front stops and the rupture termination front then propagates back from the fault edge (MADARIAGA, 1976). In this case, the healing front coincides with the rupture termination front, and is due to some dynamic wave effect. An attractive feature of this model is that the final static fault slip is a direct function of the dynamic stress drop and fault size, thus the static stress drop is proportional to the dynamic stress drop. On the other hand, the traditional seismological view, i.e., the Haskell rupture model, is that the fault heals before the rupture termination front propagates back from the furthest fault edge. HEATON (1990) discusses some of the attractive features of this type of model, which he termed the "slip-pulse" model. This model implies that there is some friction constitutive law that causes the fault to heal when the slip velocity falls to some value (ZHENG and RICE, 1998). Friction physics studies do find this type of friction constitutive law (see discussion in ScHOLZ, 1990). Unfortunately, inclusion of these complicated friction laws require a full dynamic numerical calculation just to produce a single STF. These full-dynamic stress drop models do not produce useful analytical solutions that can be used to interpret observations.

\subsection{Dynamic Rupture Models}

Dynamic rupture models specify the history of both rupture and healing fronts, and use some analytical description of the space-time slip velocity function. This $a$ priori description circumvents the calculation of a full-dynamic wave solution for every case. With the simple but reasonable choices of constant velocity rupture and healing fronts, it is possible to complete the integrals and produce analytical formulas for the observed STFs. The key advantage is for the inverse problem whereby the observed STFs immediately constrain some combination of model parameters. Some examples of dynamic rupture models are reviewed in AKI and Richards (1980), with the circular model of SATO and HiRASAwA (1973) of particular interest. This model uses a clever space-time dependence for the slip function such that at any particular time during rupture, the cumulative fault slip across the current fault size is exactly compatible with a constant static stress drop on a circular fault; and this static stress drop equals the dynamic stress drop across the fault edge. The only negative aspect of this model is that fault slip stops at exactly the same time over the entire fault. In other words, the healing front has an infinite velocity. BOATWRIGHT (1980) extended this model by including a more 
realistic crack healing front that propagates back from the edge; hence slip continues in the fault center until the healing front arrives. One consequence is that the static stress drop is now greater than the dynamic stress drop due to "extra" slip in the fault center. Since all crack models with healing fronts that propagate back from the fault edge share this same consequence, there is no need to retain the special space-time slip history of SATO and HiRASAwA (1973). In the crack and slip-pulse models that follow, I use the basic $\left(t^{-1 / 2}\right)$ slip velocity function.

\subsection{Crack and Slip-pulse Dynamic Models}

The crack model is depicted in the left side of Figure 2, while the slip-pulse model is shown at right. Both models use the same basic $\left(t^{-1 / 2}\right)$ slip velocity function, and they also share the same rupture front description which arbitrarily stops when it expands to radius, $R$. The key difference is the healing front. In the crack model, the healing front propagates back from the circular fault edge with the same velocity as the rupture front. In the slip-pulse model, the healing front starts at the fault center, then propagates out at the shear wave velocity; the duration of slip at $r=0$ is chosen such that the healing front catches the rupture front at $r=R$. With these simple prescriptions for the healing fronts, there are no new parameters introduced. That is, $V r, R$, and $\Delta \sigma_{d y}$ are the only earthquake parameters. For both the crack and slip-pulse models, the slip velocity function has the same functional form and starts at the same time:

$$
\dot{D}(t, r)=\dot{D}\left(t, t^{\prime}\right)=\frac{c v \sqrt{\tau}}{\left(t-t^{\prime}\right)^{1 / 2}}\left[H\left(t-t^{\prime}\right)-H(t-X)\right]
$$

where $t^{\prime}$ is the rupture delay time, $t^{\prime}=r / V r, H(t)$ is the step function, $v$ is a characteristic slip velocity, e.g., the Brune value, $c \sqrt{\tau}$ is a scaling constant, and $H(t-X)$ refers to the healing front, where $X$ is clearly different for the crack or slip-pulse model. From Figure 2, it is easily seen that for the crack model, $H(t-X)$ becomes: $H(t-(2 R-r) / V r)$, and for the slip pulse model, $H(t-X)$ becomes: $H(t-(R(1 / V r-1 / \beta)+r / \beta))$. We first need to connect the rupture models to the static stress drop. Integrate the crack model description to obtain the final static slip over the final circular fault:

$$
D_{\text {crack }}(r)=c v 2 \sqrt{2 \tau} t_{R}^{1 / 2} \sqrt{1-r / R}
$$

where $t_{R}=R / V r$ is the fault rupture time. Set the scaling product as follows:

$$
c v \sqrt{\tau}=\frac{24}{\sqrt{27 \pi}} \frac{\Delta \sigma_{d y}}{\mu} V r \sqrt{t_{R}}
$$

to yield the desired "overshoot" scaling between the static and dynamic stress drop: 


$$
\Delta \sigma_{s t}=\frac{8}{5} \Delta \sigma_{d y} \quad(\mathrm{Crack})
$$

The final static slip for the slip-pulse model is:

$$
D_{\text {slip-pulse }}(r)=c v \sqrt{\tau} 2 \sqrt{(1-f)} t_{R}^{1 / 2} \sqrt{1-r / R}
$$

where $f=V r / \beta$, as before. The final static displacements have the same functional form for the crack and slip-pulse models, but the amount of slip for the crack model will be greater by a factor of $[2 /(1-f)]^{1 / 2}$. Use the same setting for the $[c v \sqrt{\tau}]$ factor to obtain:

$$
\Delta \sigma_{s t}=\frac{4}{5} \sqrt{2} \sqrt{(1-f)} \Delta \sigma_{d y} \quad \text { (Slip-pulse). }
$$

The slip-pulse static stress drop depends explicitly upon the rupture velocity, but is less than the dynamic stress drop for typical rupture velocities. For a fast rupture velocity, say $f=0.9$, then the connection is:

$$
\Delta \sigma_{s t}=(0.36) \Delta \sigma_{d y} \quad(\text { Slip-pulse, } f=0.9) .
$$

The source time function is given by the slip velocity integrated over the fault area:

$$
\dot{M}(t)=\mu \iint \dot{D}(t, A) d A=2 \pi \mu \int_{0}^{R} \dot{D}(t, r) r d r
$$

where $\mu$ is the shear modulus, the directivity time shifts have been ignored, and the right-most formula takes advantage of the symmetric circular rupture geometry. After solving the integrals and some algebraic manipulation, we can write the source time functions for the crack dynamic rupture model as a function of non-dimensionalized time, $\left(t / t_{R}\right)$ :

$\dot{M}(t)_{\text {crack }}=\dot{\mathfrak{M}} f\left\{\begin{array}{l}(4 / 3)\left(t / t_{R}\right)^{3 / 2} \\ (4 / 3)\left(t / t_{R}\right)^{3 / 2}-\sqrt{2\left(t / t_{R}\right)-2}\left(2\left(t / t_{R}\right)-(2 / 3)\left(2\left(t / t_{R}\right)-1\right)\right)\end{array}\right.$

where $\dot{\mathfrak{M}}$ is given by:

for $\left.1<t / t_{R}<2\right)$

$$
\dot{\mathfrak{M}}=\mu\left(\pi R^{2}\right) \frac{24 \sqrt{2}}{7 \pi} \beta \frac{\Delta \sigma_{d y}}{\mu} .
$$

The STF continues to increase until the rupture expansion stops at time $t_{R}$, while total STF duration is $\left(2 t_{R}\right)$. Note that $\dot{\mathfrak{M}}$ gives the basic amplitude scaling for the STF, and it can be viewed as the product of three factors: shear modulus, fault area, and a "Brune-type" slip velocity.

Following the same procedure for the slip-pulse model, the final formula for the STF is: 


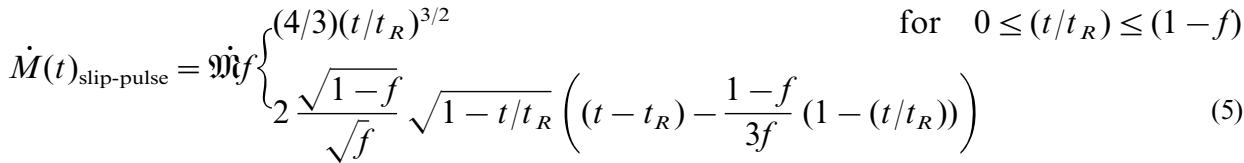

$$
\begin{aligned}
& \text { for } \quad(1-f) \leq\left(t / t_{R}\right) \leq 1
\end{aligned}
$$

where $\dot{\mathfrak{M}}$ is the same as above. Note that the total duration is just $t_{R}$ since all slip ends as soon as the rupture front reaches $R$. Thus, the peak value of the slip-pulse STF occurs before $t_{R}$, albeit with a smaller amplitude than for the crack STF. To visualize the differences in the STFs, they are plotted in Figure 3. The shapes of the STFs are quite different between the crack and slip-pulse models, thus there is some hope that observations can discriminate between these classes of rupture models.

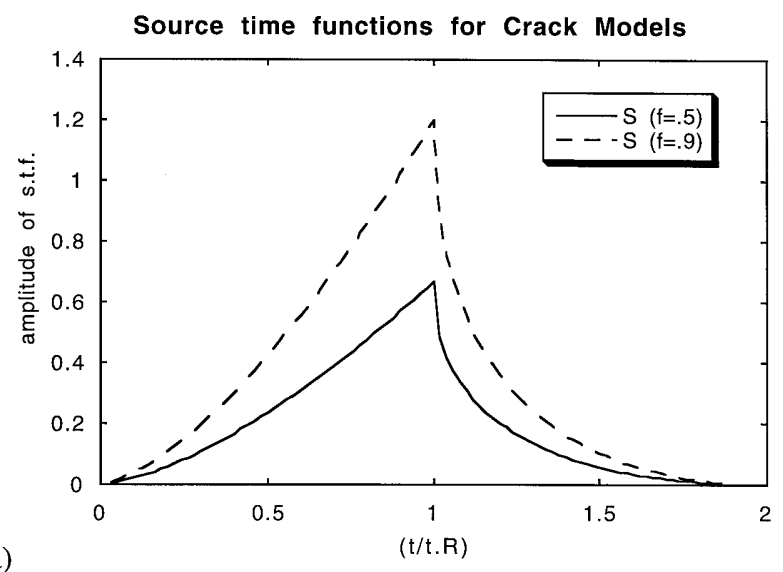

(a)

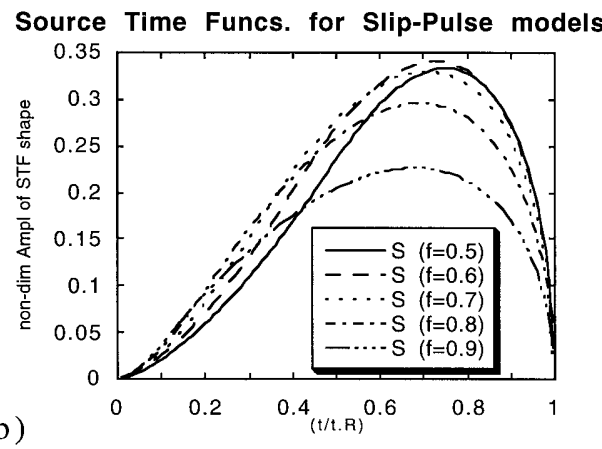

Figure 3

Theoretical source time functions for the crack (a) and slip-pulse (b) models, plotted as a function of non-dimensionalized time $\left(t / t_{R}\right)$. The amplitude scales are in the same units for (a) and (b). Several functions for different values of the non-dimensionalized rupture velocity $(f)$ are shown. The different shapes offer the possibility of finding which model better describes real earthquake rupture. 
For the crack model, the basic STF shape is the same for all values of rupture velocity when plotted as a function of non-dimensionalized time. The normalized amplitude does change with rupture velocity, STFs for two different $f$ values are shown. For the slip-pulse model, the basic shape of the STF changes for different rupture velocities, though the effect is somewhat subtle. Also note the difference in amplitude scale between the two cases. For the same fault area, dynamic stress drop, and rupture velocity, the peak amplitude of the STF will be larger for the crack model. The slip-pulse STFs reach their peak value at a time of $\left(t / t_{R}\right)=0.7$ or so. In detail, the slip-pulse STFs are the same as the crack STFs until $\left(t / t_{R}\right)$ reaches $(1-f)$. For a slow rupture velocity, such as $f=0.5$, you can see that the STF amplitude until $\left(t / t_{R}\right)=0.5$ is the same for both cases. For the slip-pulse model, the healing front begins its propagation out from the center when $\left(t / t_{R}\right)$ equals $(1-f)$, and then the slip-pulse STF falls below the still-growing crack STF.

\subsection{The Inverse Problem}

To achieve the most reliable results, I will only use the STF until the time of the initial peak value. Thus the drastic differences between the crack and slip-pulse cases after the peak are lost to us. To focus on just this initial ramp-up in the STF, there is still a different functional character between the two cases. Draw a straight line from the origin to the peak value in the STF (Fig. 3); the crack STF is always below this line while the slip-pulse STF oscillates about this straight line. In the analysis section, we will look at a few STFs with a long duration to see if it is possible to "invert" the initial shape for the rupture model.

There are two parameters that we can extract from most of the observed STFs: the peak value of moment rate, $\hat{\dot{M}}$, and time at which this peak value is obtained, $\hat{t}$. Although many of the largest earthquakes have a multiple-event STF, we can still extract these two parameters from the initial event. For the crack model case, the peak moment rate always occurs at a time of $\left(t / t_{R}\right)=1$, and its value is:

$$
\hat{\dot{M}}\left(t / t_{R}=1\right)=\dot{\mathfrak{M}} f(4 / 3) .
$$

We now rearrange the expression to solve for the dynamic stress drop:

$$
\Delta \sigma_{d y_{\text {crack }}}=\frac{C_{\text {crack }} \hat{\dot{M}}}{\beta^{3} f^{3} \hat{t}^{2}}
$$

where $C_{\text {crack }}=\left(7 /\left(2^{1 / 2} 32\right)\right)=0.155$. Note that this expression shares the same fundamental $\hat{\dot{M}} / \hat{t}^{2}$ dependence as Equation (3), though the pre-factor is different.

More work is required in the slip-pulse case to solve for the moment rate peak value and time, but the final result is similar to above:

$$
\Delta \sigma_{d y_{\text {slip-pulse }}}=\frac{S(f)}{\beta^{3} f^{3}} \frac{\hat{\dot{M}}}{\hat{t}^{2}},
$$


where $S(f)$ is given by:

$$
S(f)=(7 / 40) U(f)=(0.175)\left\{[(1+2 f) /(1-f)]^{1 / 2}[(1+f) /(1+2 f)]^{2}\right\} .
$$

The geometric scaling function $U(f)$ is of order 1 as it only varies from 1.125 for $f=0.5$ to 2.44 for $f=0.9$, whereas the $\left(1 / f^{3}\right)$ factor in Equations (6) and (7) varies quite strongly with $f$.

The above results show that the dynamic stress drop estimate depends quite strongly on the assumed value for the rupture velocity, but there is surprisingly little difference between the estimates for the crack or slip-pulse model. This latter result is a consequence of the fact that - compared to the crack model — both the peak moment rate and time to peak value decrease for the slip-pulse model such as to keep the $\hat{\dot{M}} / \hat{t}^{2}$ ratio approximately constant.

The practical consequence of this result is that our estimates of the dynamic stress drop are not critically dependent on whether we assume a crack or slip-pulse model. On the other hand, any attempt to infer a static stress drop would then require knowledge of the correct rupture model. Dynamic stress drop does have a strong dependence on $f$, but we can compare between earthquakes by assuming that $f$ is the same for all events. The value for $f$ that $\mathrm{I}$ have used in 0.7 ; for shallow earthquakes, this translates into a rupture velocity of about $2.7 \mathrm{~km} / \mathrm{s}$, in the middle of the observed range of rupture velocities. For this choice of $f$, Equations (6) and (7) become:

$$
\Delta \sigma_{d y_{\text {crack }}}(f=0.7)=\frac{0.452}{\beta^{3}} \frac{\hat{\dot{M}}}{\hat{t}^{2}},
$$

and

$$
\Delta \sigma_{d y_{\text {slip-pulse }}}(f=0.7)=\frac{0.725}{\beta^{3}} \frac{\hat{\dot{M}}}{\hat{t}^{2}} .
$$

Both model estimates are within a factor of two of each other, thus we can just calculate one model-independent estimate that is close to the average of the above:

$$
\Delta \sigma_{d y}(f=0.7)=\frac{0.575}{\beta^{3}} \frac{\hat{\dot{M}}}{\hat{t}^{2}} .
$$

In preparation for our analysis of observations, assign SI units to the above quantities. Since our STF inversions for the shallow subduction earthquakes use $3.86 \mathrm{~km} / \mathrm{s}$ for $\beta,\left(1 / \beta^{3}\right)$ has a value of $\left(5.75 \times 10^{10} \mathrm{~m}^{3} / \mathrm{s}^{3}\right)^{-1}$. Thus we have:

$$
\Delta \sigma_{d y}(f=0.7)=\frac{\hat{\dot{M}}}{\hat{t}^{2}} \times 10^{9} \mathrm{~Pa},
$$

where the units of $\hat{\dot{M}}$ are $10^{20} \mathrm{Nm} / \mathrm{s}$ and $\hat{t}$ is in seconds. We are now ready to analyze the observed STFs. 


\section{Analysis of Source Time Functions}

The Michigan STF project has systematically determined the source time functions on a near-real-time basis for global seismicity since June, 1994. TANIOKA and RUFF (1997) describe the source time function catalog and they analyze this catalog for some source time function systematics. The current catalog now includes four years of seismicity from June 1, 1994 to June 1, 1998, with a total of 190 earthquakes in the STF catalog. This catalog is globally complete for earthquakes with $M_{w} \geq 7\left(M_{0} \geq 0.3 \times 10^{20} \mathrm{Nm}\right)$ over this four-year period, and the range of earthquake size is from $M_{w}=5.6$ to 8.3. The STFs are available on the web (start at: "http://www.geo.lsa.umich.edu/SeismoObs/"), and an on-line data base will be available in 1999. To focus on the large events, the four-year catalog has 62 earthquakes with $M_{w} \geq 7$, and about 30 of these events are subduction underthrusting earthquakes. I have screened the entire STF catalog for this stress drop analysis; earthquakes are not used if the initial ramp-up in the STF is indistinct due to precursors or multiple-events. The procedure is to find the amplitude and time of the initial peak in the STFs, then use Equation (10) to calculate the dynamic stress drop. Figure 4 plots the results, where earthquakes are plotted as stress drop versus their overall seismic moment; different symbols are used for different tectonic settings. The scatter in the stress drop estimates is almost a factor of one thousand! Most studies of static stress drop systematics find a scatter of a factor of ten to one hundred (SCHOLZ, 1990), so it is a surprising result that the more reliable (see Section 4) dynamic stress drop shows even more scatter. One interpretation is that the true dynamic stress drop of earthquakes is as variable as the estimates in Figure 4 , but first lets consider other explanations. We expect that the deep slab events will be shifted to larger stress drop values because we use a crustal value for $\beta$. Substitution of the correct value for $\beta$ shifts the stress drop for deep slab events down by a factor of 3 . By comparison, any variation in $\beta$ for shallow events is minor - with the possible exception of "tsunamigenic" events that occur in the accretionary prism (SATAKE, 1995). Although $\beta$ may be much smaller within a fault zone, it is the value of $\beta$ in the volume of rock surrounding the fault that is relevant. If the rupture front geometry is a quarter-circle rather than a circle, the stress drop estimate in Figure 4 would increase by about a factor of 4 . If we look back at Equations (6) and (7), the only other source of potential scatter is $f$, the rupture velocity. Figure 4 assumes that $f$ is 0.7 for all events. If $f$ were 1 or 0.5 , then the shift in stress drop estimates would be about a factor of 3 down or up. The only way to significantly reduce the scatter would be to assign very slow rupture velocities to those events with low stress drops. In particular, to increase the stress drop from 5 to 100 bars, we need to assume that $f=0.25$, which means that $V r=1.0 \mathrm{~km} / \mathrm{s}$. For some of the larger earthquakes with low stress drop estimates, we know that their rupture velocity is not as slow as $1 \mathrm{~km} / \mathrm{s}$. For the small earthquakes, some of the scatter can be explained by resolution difficulties in the 


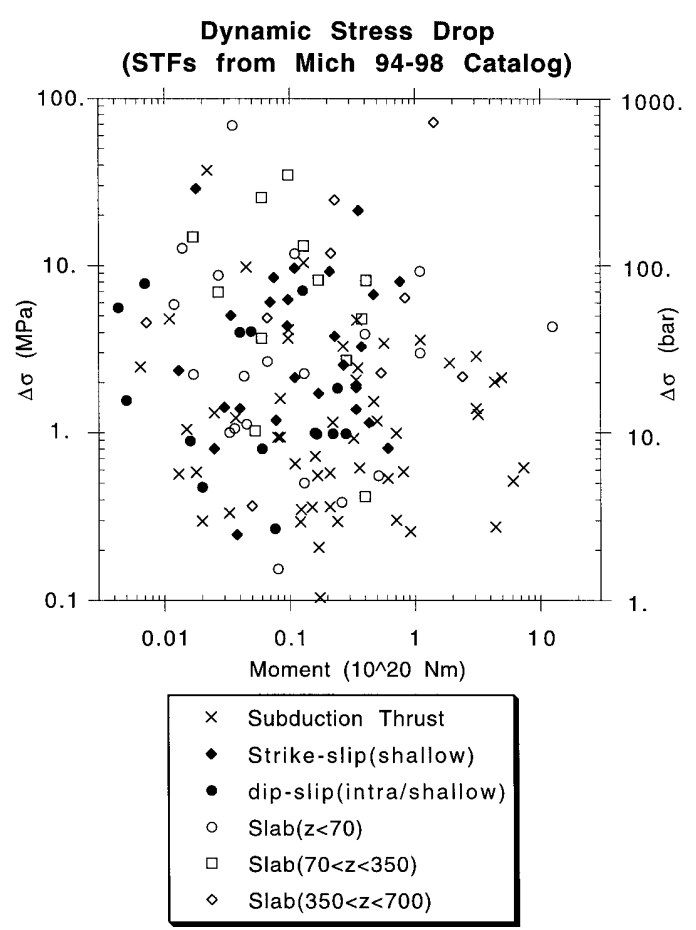

Figure 4

Dynamic stress drop for all suitable earthquakes in the Michigan STF catalog. Each point plots the dynamic stress drop estimate (MPa on left axis, bars on right axis) as a function of the total seismic moment for that event. Different symbols are used for the tectonic settings listed in legend. Note the factor of one thousand scatter. The larger subduction thrust events have stress drops between 2 and 50 bars.

temporal sampling of the STF, but this type of error is not important for the larger earthquakes. Thus, it appears that the dynamic stress drop is in fact quite variable between earthquakes, even between earthquakes of the same size that occur in the same plate boundary. This conclusion becomes even more obvious when we plot the STFs - there is no question that the earthquake parameter of $\hat{\dot{M}} / \hat{t}^{2}$ is resolvably different.

Figure 5 plots the initial part of the STFs for several of the largest shallow earthquakes; all are subduction underthrust events except for the great 941004_Kuriles event (Note: Earthquakes are identified by their occurrence data, YrMnDy, and a regional name. Hypocentral parameters can be found in the on-line STF catalog, or from many other sources.) After seeing the STF variation in Figure 5, it is not surprising that the rapid increase in moment rate for the 941004_Kuriles event translates into a dynamic stress drop estimate of 43 bars, while the slow increase in the STF for the 951009_Mexico event translates into a stress drop estimate of just 3 bars. Some large events, including the largest deep and 
shallow events in the past four years, are not plotted in Figure 4 due to some complication in the initial ramp of their STF. Figure 6 shows the STFs for the largest multiple-event subduction earthquakes, including the 960217_Irian_Jaya event which is the largest earthquake in this catalog. For these multiple-event earthquakes, it is possible to calculate a dynamic stress drop for the first subevent. These first-event stress drop estimates fall within the range of values defined by the other subduction earthquakes in Figure 4.

\subsection{Test of Rupture Model}

The STF for the great 941004_Kuriles event presents some striking evidence for the slip-pulse model as the STF oscillates about a straight line connecting the origin to the peak value. Several other large events share this qualitative feature, including the 960101_Sulawesi and 960225_Mexico events. On the other hand, there does not seem to be any large subduction earthquake with a STF that argues strongly for the crack model. Overall, most of the STFs are well-characterized by just a straight line ramp-up to the initial peak, with some minor deviations. Recall from Section 4 that the ratio of static to dynamic stress is another indicator of rupture model. Independent determinations of the static stress drop are available for several of the larger earthquakes (TANIOKA and RUFF, 1997). For the 941004_Kuriles event, the static stress drop is 70 bars, while the dynamic stress drop from Figure 4 is 43 bars. For the 951009_Mexico event, the static stress drop is 4 bars, while the dynamic stress drop is 3 bars. For the 941228_Sanriku event, the static and initial dynamic

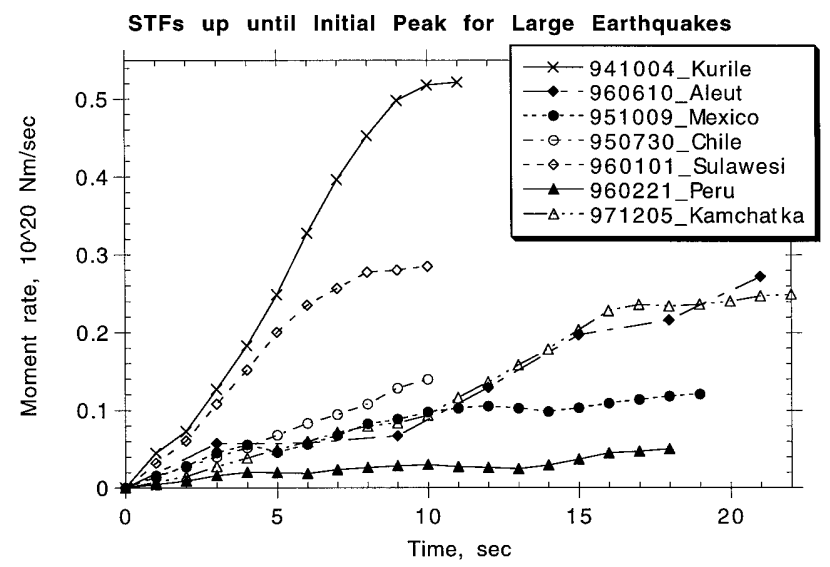

Figure 5

Source time functions (STFs) for seven of the largest earthquakes where the initial peak in the STF is the largest peak for the entire STF. All STFs use the same amplitude scale (units are $\times 10^{20} \mathrm{Nm} / \mathrm{s}$ ). The STFs are plotted only up until the time of their peak value. Earthquakes are identified by date and region, i.e., 941004_Kurile is the October 4, 1994 great event in the Kuriles subduction zone. STFs that reach a higher peak amplitude at a shorter time will have larger dynamic stress drops. 


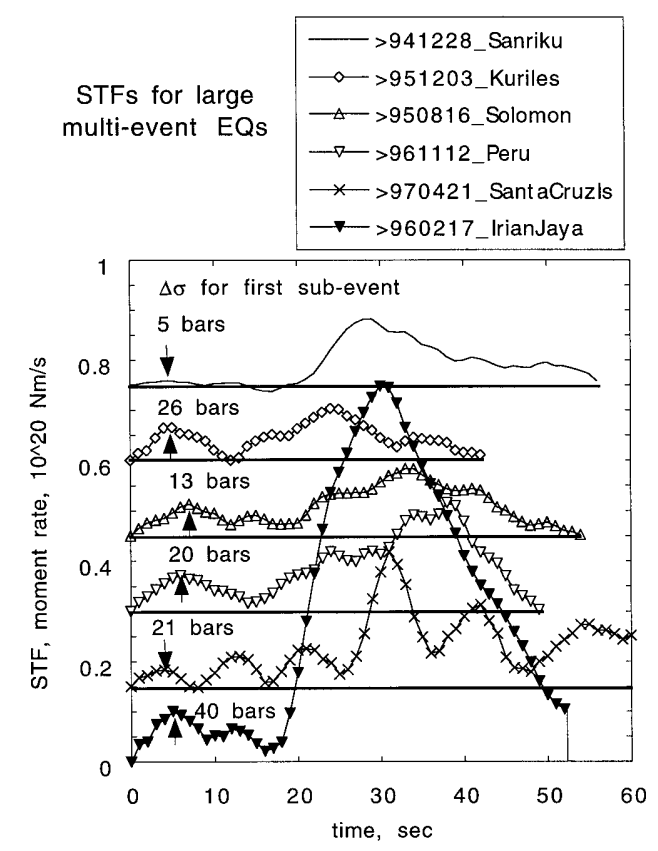

Figure 6

Complete STFs for six of the largest multiple-event earthquakes of the past four years. The baselines are shifted for each event for plotting clarity, but they all use the same amplitude scale. The total moment release in the first event is a small fraction of the final seismic moment for these events. The dynamic rupture models are only reliable up until the time of the initial peak in the first events. Stress drop estimates based on the initial peak values are shown for each earthquake.

stress drops are 6 and 5 bars, respectively. From this limited comparison, it seems that the ratio of static to dynamic stress drop is about $\sim 1$, and hence we cannot discriminate between the crack or slip-pulse models. To conclude, this brief look at the STFs does not indicate a compelling preference for either the crack or slip-pulse model, though there are a few clear examples of the STF shape expected from the slip-pulse model.

\subsection{The Seismogenic Interface in Subduction Zones}

Underthrusting earthquakes in subduction zones occur on the rather narrow seismogenic portion of the plate interface. The down-dip edge of the seismogenic zone occurs at a depth of about $40 \mathrm{~km}$ for many of the world's subduction zones, though it does show some variation that appears to be correlated with the thermal state of the subduction zone (see TICHELAAR and RUFF, 1993). As part of their thermal modeling, TICHELAAR and RUfF (1993) found the best overall global description of frictional shear stress through the seismogenic zone. Their estimates are quite uncertain due to sparse heat flow observations and model uncertainties. 
TICHELAAR and RUFF (1993) tested two shear stress distributions: constant stress; and constant friction coefficient which yields a linear increase in stress through the seismogenic zone. For the constant shear stress experiment, they find a range in values from 14 to $43 \mathrm{MPa}$ for the individual subduction zones. For the constant friction coefficient experiment, they find a range in values from 0.047 to 0.127 . The global median value is 0.059 , with a $90 \%$ confidence interval from 0.044 to 0.065 . TICHELAAR and RUFF (1993) preferred the constant friction coefficient case due to characteristics of the resultant geotherms. Regardless of exactly which model is used, we see that the absolute shear stresses are likely to be more than $10 \mathrm{MPa}$ at the down-dip edge of the seismogenic zone. I use the preferred model of TICHELAAR and RUFF (1993) that has the shear stress increasing linearly from zero at the trench to a value that ranges from 46 to $68 \mathrm{MPa}$ at a depth of $40 \mathrm{~km}$.

The up-dip edge of the seismogenic zone is not as well-constrained from seismological observations. In some places, there is a distinct up-dip edge, while in other places it seems that the seismogenic zone extends up to the trench. While the up-dip edge may be sharply defined in some places by small earthquakes, it is possible that the rupture front from a great earthquake may propagate up beyond this edge. The study of the northern Honshu subduction zone by TANIOKA et al. (1997) show that there might be two different environments for the plate interface near the trench and that unusual tsunamigenic earthquakes can occur in the uppermost plate interface zone that is usually thought to be aseismic. Thus, there are many puzzles related to the up-dip portion of the seismogenic interface. Here, I will focus on just one aspect of the seismogenic zone: is there a variation in stress drop for earthquakes that initiate at the up-dip edge versus those that initiate at the down-dip edge? This question is prompted by some of the unusual characteristics of the 941228_Sanriku earthquake. In particular, its epicenter is at the up-dip of the seismogenic zone, and its initial dynamic stress estimate is low, about 5 bars (see Fig. 7). Smaller events that have occurred at the down-dip edge in Sanriku have larger values for stress drop (e.g., the 950106_Sanriku aftershock, $M_{w}=6.7$, has a dynamic stress drop of about 100 bars). Earlier studies have also suggested that underthrusting events located closer to the trench may have lower stress drops (FukaO, 1979; Schwartz and RufF, 1987).

There is one subduction zone segment that is particularly well-sampled by the STF catalog: the Oaxaca segment of the Mexico subduction zone. Figure 8 shows the STFs of six Mexico underthrusting events together with a schematic epicenter map for the four events along the Oaxaca coast. Four Mexico earthquakes have STFs and stress drops that fall in the typical middle range for Mexico, e.g., the 960225 event has a dynamic stress drop of 5 bars. However, the 950914 event has a higher value of 36 bars, while the 970719 event has a lower value of 1 bar. Figure 8 shows that these STFs are resolvably different from the others. Notice that the epicenter of the 950914 event places it at the down-dip edge of the seismogenic zone, while the 970719 event is at or near the up-dip edge. To make the stress drop 
estimate of the 970719 event as large as the 950914 event, we would have to say that its rupture velocity was only $0.8 \mathrm{~km} / \mathrm{s}$. Although we cannot uniquely unravel the contributions of dynamic stress drop and rupture velocity, there is a clear systematic gradient in the earthquake rupture process across the seismogenic interface. The stress drop and/or rupture velocity systematically increase from the up-dip to down-dip edges in the Oaxaca segment of this subduction zone.

\section{Conclusions}

The crack and slip-pulse dynamic rupture models differ only in the behavior of the healing front, yet they produce source time functions that look quite different. However, the inverse problem for an estimate of the dynamic stress drop based on the initial ramp-up of source time functions is NOT sensitive to the choice of rupture model as long as the rupture velocity is a typical value. The Michigan STF catalog is processed to obtain dynamic stress drops for global seismicity of the past
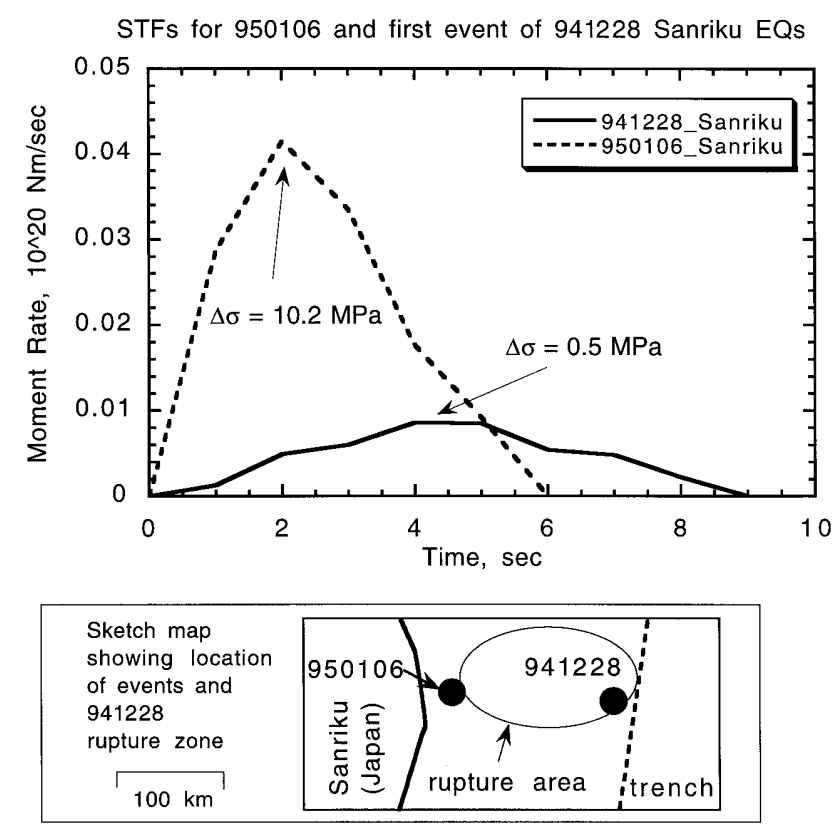

Figure 7

Initial part of STFs for two Sanriku subduction events (upper), and a schematic epicenter map that shows the relative positions of the two events and the 941228 rupture zone along the Sanriku subduction segment (lower). The variation in the initial "up" of the STFs translates into significant variation in the stress drop estimates (also shown). The 941228 epicenter is quite close to the trench, and it has a very small value for stress drop of the initial rupture. The small event at the down-dip edge of the seismogenic zone has a high value for dynamic stress drop. 


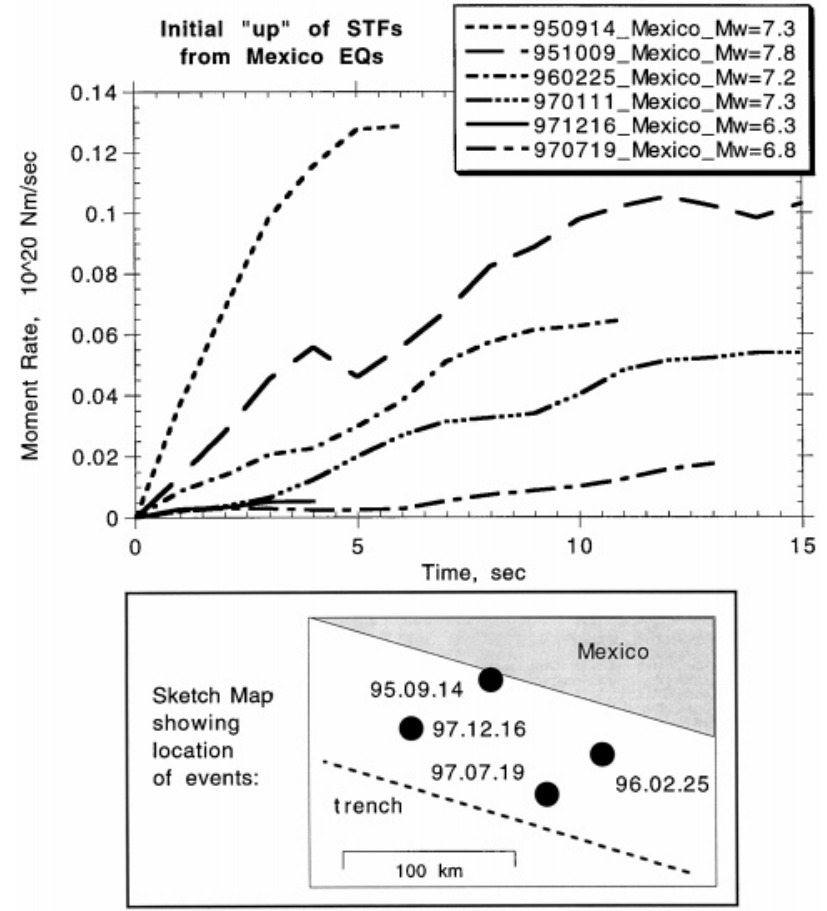

Figure 8

Initial part of STFs for Mexico subduction events (upper), and a schematic epicenter map that shows the relative positions of four events that occur close together along the Oaxaca segment (lower). The variations in the STFs translate into significant variations in the stress drop estimates. The event closest to the trench (970719) has a very small value for stress drop, while the event at the down-dip edge of the seismogenic zone (950914) has a high value for stress drop. The other events have intermediate stress drop values.

four years, and the first conclusion is that the scatter in dynamic stress drop is almost a factor of one thousand. There may be some coherent structure in this scatter. In particular, a profile of events that span the seismogenic interface in the Mexico subduction zone show a systematic increase in stress drop from the up-dip to down-dip edges of the seismogenic zone. At this time, there is no other subduction zone segment as well sampled as Mexico.

These preliminary results permit some speculation about the stress state of the seismogenic zone. Figure 9 sketches the scenario that emerges from the study of TICHELAAR and RUfF (1993) on the absolute level of shear stress and from the results presented here. Stress drops for some of the smaller underthrust events are $10 \mathrm{MPa}$ (e.g., the 950106 Sanriku event), but values for the larger underthrust events $\left(M_{0}>10^{19} \mathrm{Nm}\right)$ at the down-dip edge of the seismogenic zone do not exceed a value of $5 \mathrm{MPa}$. Thus, I propose that the "typical" dynamic stress drops at the up-dip and down-dip edges of the seismogenic zone are 0.2 and $5 \mathrm{MPa}$, respectively. 
If we make the rash assumption that we can combine these dynamic stress drops with the frictional shear stress result of TICHELAAR and RUFF (1993), then we must conclude that the stress drop of large subduction earthquakes at the down-dip edge is just a partial stress drop. Based on the earlier discussion of the absolute shear stress estimates, I show a shear stress of about $50 \mathrm{MPa}$ in Figure 9 the down-dip edge of the seismogenic zone; a mid-range value from the TICHELAAR and RUFF (1993) results. The low dynamic stress drops for events at the up-dip edge would explain the occasional occurrence of so-called "slow earthquakes" near the trench; the low value of dynamic stress drop causes a slow increase in the first event of the source time function. It is curious that the increase in dynamic stress drop from the

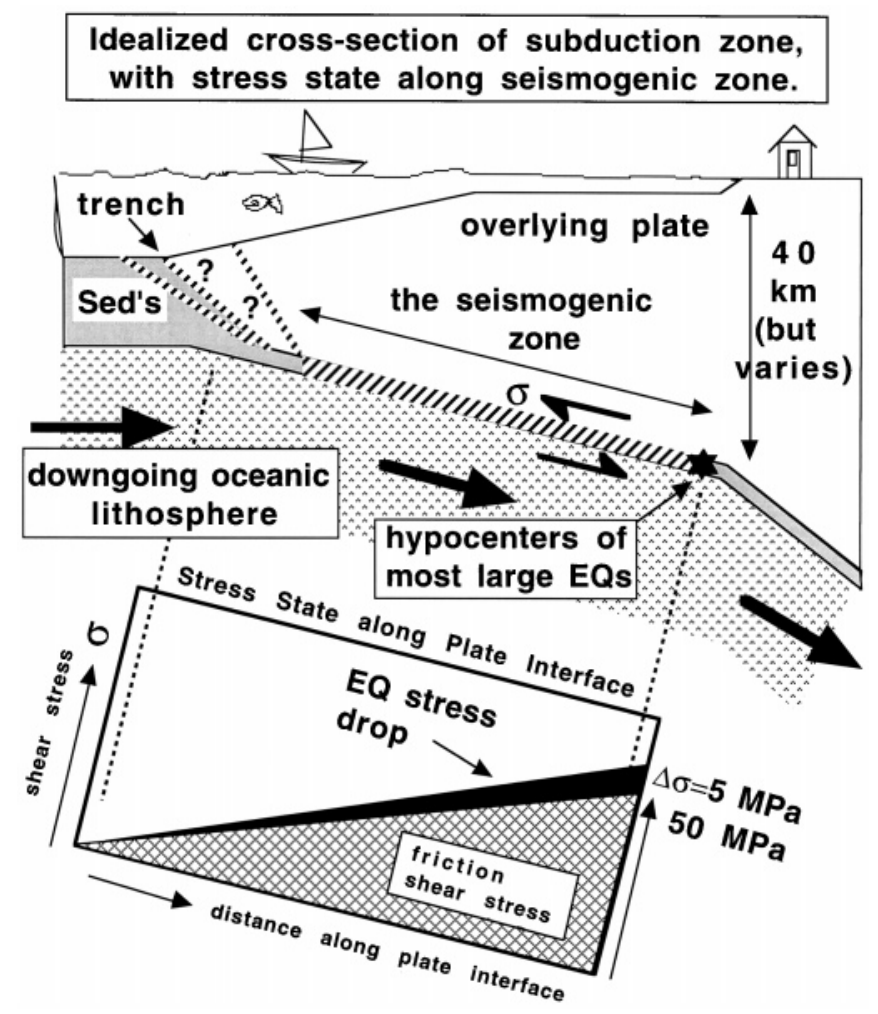

Figure 9

Speculations on the stress state within the seismogenic zone of a "typical" subduction zone that generates large underthrusting earthquakes. The linearly increasing friction shear stress is based on the modeling of TICHELAAR and RUfF (1993), and reaches a peak value of $50 \mathrm{MPa}$ at the down-dip edge. Based on the results of this paper - which are broadly compatible with some previous results - earthquake stress drops also increase with depth along the seismogenic zone. The analysis of the large underthrusting events in the past four years suggests that dynamic stress drops are about $0.2 \mathrm{MPa}$ at the up-dip edge and $5 \mathrm{MPa}$ at the down-dip edge of the seismogenic zone. If this speculative scenario is correct, then the earthquake rupture process of large earthquakes only lowers the shear stress by about $10 \%$ of its absolute value. 
up-dip to the down-dip portions of the seismogenic zone mimics the gradient in friction shear stress. One implication of the stress state shown in Figure 9 is that most of the earthquake energy release goes into friction.

Do all subduction zones show the same systematic variation in earthquake rupture as seen in Sanriku and Mexico? Is it possible that this pattern might change within the same subduction zone segment over the seismic cycle? The current STF catalog cannot answer these questions at this time, but perhaps future studies can add more examples to this intriguing picture of the stress variations within the seismogenic zone.

\section{Acknowledgments}

Thanks to all my colleagues and students who have participated in the Michigan STF project over the last four years. The reviewers and editor made several helpful suggestions. This project is partly supported by the National Science Foundation (EAR-9725175).

\section{REFERENCES}

Aki, K., and Richards, P. G., Quantitative Seismology (W. H. Freeman, San Francisco 1980) 932 pp. Bontwright, J. (1980), A Spectral Theory for Circular Seismic Source; Simple Estimates of Dimension, Dynamic Stress Drop, and Radiated Energy, Bull Seismol. Soc. Am. 70, 1-27.

Brune, J. N. (1970), Tectonic Stress and the Spectra of Seismic Shear Waves from Earthquakes, J. Geophys. Res. 75, 4997-5009.

FukAO, Y. (1979), Tsunami Earthquakes and Subduction Processes near Deep-sea Trenches, J. Geophys. Res. 84, 2303-2314.

Heaton, T. (1990), Evidence for and Implications of Self-healing Pulses of Slip in Earthquake Rupture, Phys. Earth and Planet. Int. 8, 25-83.

Kanamori, H., and Anderson, D. L. (1975), Theoretical Basis of Some Empirical Relations in Seismology, Bull. Seismol. Soc. Am. 65, 1073-1095.

Kasahara, K., Earthquake Mechanics (Cambridge University Press, Cambridge 1981) 248 pp.

Madariaga, R. (1976), Dynamics of an Expanding Circular Fault, Bull. Seismol. Soc. Am. 66, 639-666.

Ruff, L. J., and Kanamori, H. (1983), The Rupture Process and Asperity Distribution of Three Great Earthquakes from Long-period Diffracted P Waves, Phys. Earth Planet. Int. 31, 202-230.

SATAKE, K. (1995), Linear and Nonlinear Computations of the 1992 Nicaragua Earthquake Tsunami, Pure appl. geophys. 144, 455-470.

Sato, T., and Hirasawa, T. (1973), Body Wave Spectra from Propagating Shear Cracks, J. Phys. Earth $23,323-331$.

SchOlz, C. H., The Mechanics of Earthquakes and Faulting (Cambridge University Press, Cambridge 1990) $439 \mathrm{pp}$.

Schwartz, S. Y., and Ruff, L. J. (1987), Asperity Distribution and Earthquake Occurrence in the Southern Kuril Islands Arc, Phys. Earth Planet. Int. 49, 54-77.

Tanioka, Y., and Ruff, L. J. (1997), Source-time Functions, Seismol. Res. Lett. 68, 386-400.

Tanioka, Y., Ruff, L. J., and Satake, K. (1997), What Controls the Lateral Variation of Large Earthquake Occurrence along the Japan Trench? The Island Arc 6, 261-266. 
TichelaAr, B., and Ruff, L. J. (1993), Depth of Seismic Coupling along Subduction Zones, J. Geophys. Res. 98, 2017-2037.

Zheng, Z., and Rice, J. R. (1998), Conditions under which Velocity Weakening Friction Allows a Salf-healing Versus a Crack-like Mode of Rupture, Bull. Seismol. Soc. Am. 88, 1466-1483.

(Received July 15, 1998, revised/accepted February 4, 1999) 